\title{
Giardia lamblia's Ventral Disc Microtubules Transition Through as Many as Six Structurally Distinct Regions
}

\author{
Cindi L. Schwartz ${ }^{1}$, Joanna R. Brown ${ }^{1}$, John M. Heumann ${ }^{1}$, Scott C. Dawson ${ }^{2}$, and Andreas Hoenger ${ }^{1}$ \\ ${ }^{1}$ University of Colorado, Boulder Lab for 3-D Electron Microscopy of Cells, Dept. MCD Biology, \\ Boulder, CO, 80309, USA. \\ ${ }^{2}$ University of California Davis, Dept. Microbiology, Davis, CA, 95616, USA.
}

Giardia lamblia is a unicellular intestinal parasite of animals that has evolved a unique microtubule (MT) cytoskeleton critical for pathogenesis; 4 pairs of flagella, the median body, and the ventral disc (VD). The VD is a large, highly regular spiral array (Fig. 1A) of MTs and associated microribbons (MR) [1]. MRs are unique to Giardia species and consist of three lamellar sheets that extend dorsally from the MTs into the cytoplasm and are connected laterally to neighboring MRs by crossbridges [2]. We have used tomography (Fig. 1B) to determine the disc is formed from $95+/-5$ evenly spaced, parallel MTs, adding up to a total length of $1.23+/-0.06 \mathrm{~mm}$, doubling previous estimates [3].

Recent work identified more than thirty disc-associated proteins (DAPs) on the ventral disc [4]. However, only the specific localization (MT or MR) of a few DAPs is known [5]. Many GFP-tagged DAPs do not localize uniformly across the disc, but are restricted to particular regions [4]. These regions correlate well with structural and organizational differences we can observe in both negatively stained (Fig. 1B-D) and frozen-hydrated isolated VDs (Fig. 1E). We now define six structurally distinct regions in the disc: dense bands, dorsal overlap zone, disc body, ventral groove, ventral overlap zone, and disc margin (Fig. 1C). These regions are not composed of separate microtubules. Instead, individual microtubules pass through as many as six regions, undergoing up to five structural and organizational transitions (Fig. 1D). The type and arrangement of the DAPs also alters along the length of a single microtubule, with the type and complexity of decoration varying significantly (Fig. 1E).

Using cryo-electron tomography of isolated cytoskeletons (CS) followed by subvolume averaging, we have obtained averages from the disc body [1], disc margin, and both layers of the overlap zone (Fig. 1E). This study revealed a surprising amount variation in the structural details between these regions that has been undetected using other microscopic methods. It not only demonstrates the power of these techniques, but also supports that various disc regions and protein complexes may have differential contributions to the overall function of the disc. Further our work implies a highly regulated and complex assembly of the VD that has yet to be discovered [7].

\section{References:}

[1] CL Schwartz, JM Heumann, SC Dawson, A Hoenger. PLoS ONE 7(9) (2012).

[2] DV Holberton. JCS 13 (1973), 11-41.

[3] DV Holberton, AP Ward. JCS 47 (1981), 139-166.

[4] K Hagen, M Hirakawa, S House, CL Schwartz, et al. PLoS Neg Trop Dis 5 (2011).

[5] D Macarisin, C O’Brien, R Fayer, G Bauchan, M Jenkins. Parasitol Res 111 (2012), 241-248.

[6] W Denk, H Horstmann. PLoS Biol 2(11) (2004).

[7] Electron tomography was performed in the Boulder Laboratory for 3D Electron Microscopy of

Cells, supported by P41GM103431 from the NIGMS to A. Hoenger. 

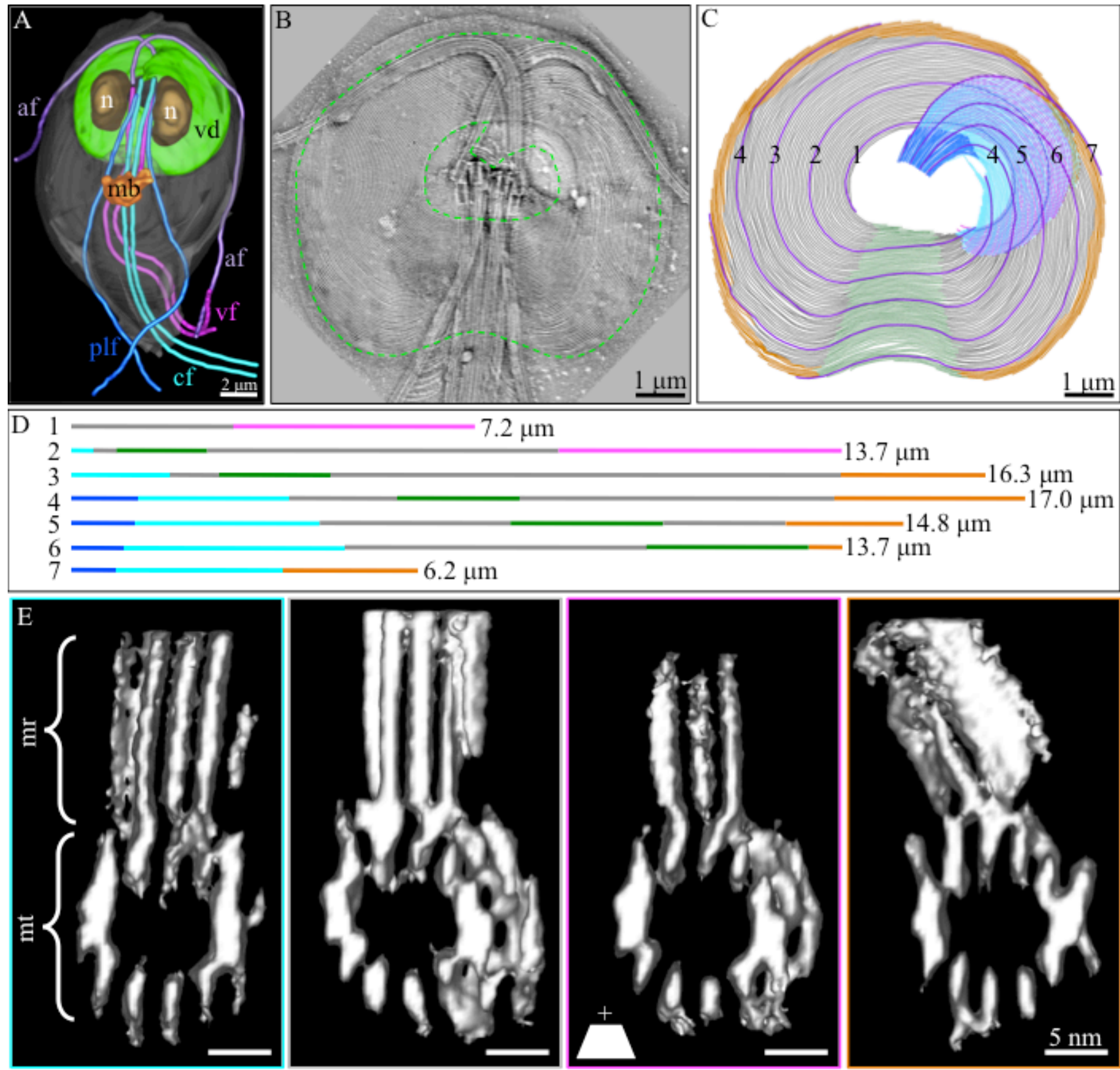

Figure 1. Giardia's VD is a complex MT array of distinct structural regions. A) Block-face imaging $[1,6]$ reveals a binucleate (n) cell with a CS consisting of the ventral disc (vd), median body (mb), and flagella; anterior (af), caudal (cf), posterior-lateral (plf), and ventral (vf). B) A negatively stained CS imaged with large-area tomography (VD in green). C) Each MT is shown. Regions are colored based on morphology and DAP localization [4]; dense bands (blue), dorsal overlap zone (cyan), disc body (grey), ventral groove (green), ventral overlap zone (magenta), and disc margin (orange). 7 representative microtubules are purple. D) The seven MTs, where proportional lengths of regions are indicated by color. The total length of each MT is noted. E) Volume averages from cryo-electron tomograms of four VD regions. Each average consists of a microtubule $(\mathrm{mt})$ with attached microribbon (mr). There are clear differences between each region; dorsal overlap zone (left, cyan), disc body (left middle, grey), ventral overlap zone (right middle, magenta), disc margin (right, orange). Polarity is noted. 